\title{
Acute Myocardial Infarction Caused by Tumor-Associated Thrombotic Thrombocytopenic Purpura: Case Report
}

\author{
Jun Wang ${ }^{\mathrm{a}}{\text { Xiaomin } \mathrm{Cai}^{\mathrm{a}} \text { Xunmin Cheng }{ }^{\mathrm{a}} \text { Ping Song }^{\mathrm{b}} \text { Shisen Jiang }}^{\mathrm{a}}$ \\ Jianbin Gong ${ }^{\text {a }}$ \\ Departments of a Cardiology and ${ }^{b}$ Hematology, School of Medicine, Nanjing University, Jinling Hospital/Nanjing \\ General Hospital of Nanjing Military Command, Nanjing, China
}

\section{Key Words}

Myocardial infarction - Thrombotic thrombocytopenic purpura $\cdot$ Gastric carcinoma

\begin{abstract}
Objective: To describe a case with acute myocardial infarction caused by gastric carcinoma-associated thrombotic thrombocytopenic purpura. Clinical Presentation and Intervention: A 79-year-old man was admitted with abdominal pain and pyrexia. He later developed cardiac complications and microangiopathy that indicated worsening progression. Manifold evidence confirmed the diagnosis of myocardial infarction caused by thrombotic thrombocytopenic purpura. The patient was treated mainly with plasma transfusion incorporated with steroids. Conclusion: This case should remind physicians to consider microangiopathy as a differential diagnosis in patients with unexplained cardiac symptoms or atypical presentation. Early diagnosis and treatment are helpful in decreasing the sequelae of this syndrome.

(c) 2013 S. Karger AG, Basel
\end{abstract}

\section{Introduction}

Thrombotic thrombocytopenic purpura (TTP) is an uncommon syndrome. The primary TTP is agitated by a disintegrin and metalloproteinase with a thrombospondin type 1 motif, member 13 (ADAMTS13) deficiency which drives the process of von Willebrand factor (vWF) accumulation, platelet aggregation, hyaline microthrombosis and eventually microvascular occlusion. Since the first case reported by Moschcowitz in 1925 [1], a few secondary cases with cardiac involvement has been reported [2]. We report on a patient with TTP who presented with acute myocardial infarction (MI).

\section{Case Report}

A 79-year-old man was admitted to the Department of Gastroenterology with the main complaint of abdominal pain and pyrexia. Physical examination did not reveal any specific signs. Electrocardiogram was within the normal range. Gastroscopy revealed a villous adenoma (high-grade intraepithelial neoplasia) and deteriorated erosion in the corpus ventriculi. Preliminary laboratory findings revealed a threshold of renal dysfunction, a moderately increased level of lactate dehydrogenase and tumor marker CA125. After 2 days, the situation was unexpectedly aggravated by noctur-

Dr. Jianbin Gong

Department of Cardiology, School of Medicine, Nanjing University Jinling Hospital/Nanjing General Hospital of Nanjing Military Command 305 Zhongshan East Road, Nanjing 210002 (China)

E-Mail zero991127@ hotmail.com
This is an Open Access article licensed under the terms of the Creative Commons Attribution-NonCommercial 3.0 Unported license (CC BY-NC) (www.karger.com/OA-license), applicable to the online version of the article only. Distribution permitted for non-commercial purposes only. 
Fig. 1. The comparison of electocardiograms from $\mathrm{A}$, the period of morbidity before the incidence, and from $B$, after the incidence.

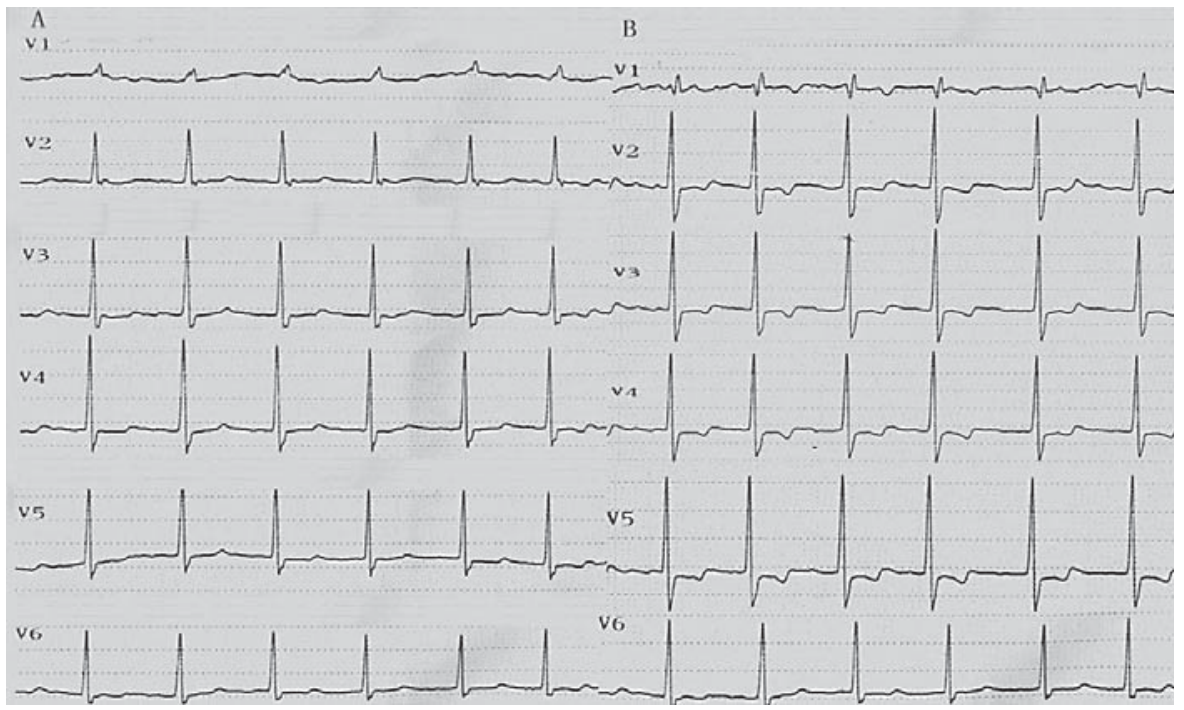

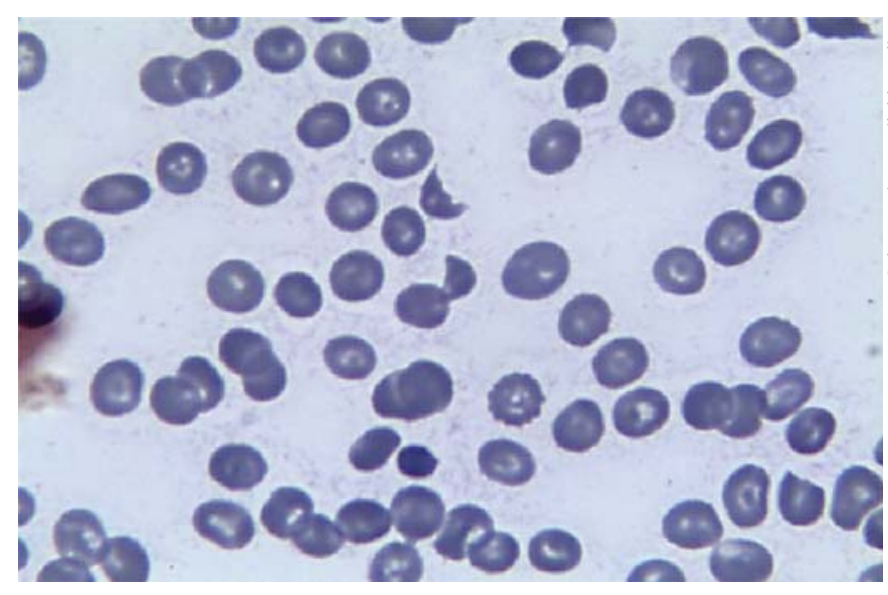

Fig. 2. Schistocytes displayed in the peripheral blood smear. Wright-Giemsa. $\times 100$.

nal dyspnea and hyperpyrexia, including signs of dysphoria, perspiration, orthopnea and pulmonary moist rales. Arterial pressure was 123/89 mm Hg. Emergent blood analysis showed an upsurge of indirect bilirubin, hepatic enzyme and myocardial enzyme as well as creatinine. Moreover, leukocytes began to increase, while platelets declined. Electrocardiogram indicated a rapid AF and notable ST-T depression (fig. 1). The echocardiography revealed a low ejection fraction of $42 \%$ (normal value: $50 \%$ ). In view of suspicion of myocardial infarction and myocardial dysfunction, the standard treatment was given i.e. diuretics (furosemide), vasodilation (sodium nitroprusside), ventricular rate control (lanatoside C) and a beta blocker. The patient was later transferred to the Coronary Care Unit.

Soon afterwards, the cardiologists noted an alteration in the patient's appearance, with haziness, dysphoria or coma being ob- served. He occasionally complained of headache, fatigue or nausea. Delirium, petechiae and mild jaundice were exhibited successively. There was a progressively increased trend of hepatic enzyme, bilirubin and creatinine. Laboratory tests showed that the comprehensive myocardial enzymes release curve matched with a typical MI. Thereafter, the cardiologists observed that the hematocytes diminished (except leukocytes which increased), erythrocytes reduced to $2.93 \times 10^{12} / \mathrm{l}$, hemoglobin to $86 \mathrm{mg} / \mathrm{dl}$ and the platelet count to $8 \times 10^{9} / 1$ at a minimum. Moderate occult blood was detected in routine urinalysis. The peripheral blood smear showed schistocytes (fig. 2). The hematology laboratory was unable to detect the activity of vWF and ADAMTS13. Chest X-ray suggested pulmonary congestion and possible pneumonedema. Cranial computed tomography scanning was negative. We logically proposed the final diagnosis of 'tumor-associated TTP complicated by acute non-ST-segment elevation myocardial infarction'. As a result, the patient was treated with regular plasma transfusion in collaboration with steroids (methylprednisolone) and continuous renal replacement therapy.

The general condition of the patient improved remarkably. Consciousness was regained and symptoms were relieved. The biochemical parameters were restored: the platelet count $150 \times$ $10^{9} / \mathrm{l}$, erythrocytes $2.2-2.3 \times 10^{12} / \mathrm{l}$ and hemoglobin $70 \mathrm{mg} / \mathrm{dl}$, the myocardial enzymes attenuated and creatinine fluctuated within the range of $200-400 \mu \mathrm{mol} / \mathrm{l}$. After 20 days in intensive care, the patient was discharged but depended on periodical continuous renal replacement therapy. At 1-month follow-up, the illness was stable.

\section{Discussion}

We illustrated a patient who presented with TTP complicated with cardiovascular injury. TTP is the microangiopathic syndrome characterized by hemolytic anemia, 
thrombocytopenia, neurological disorders, renal dysfunction and fever, as in this case. Cardiac manifestations are typically silent. Diagnosis was made on the basis of clinical features, as previously reported [3].

ADAMTS13 deficiency is responsible for the platelet thrombi in the microvasculature, the pathophysiological hallmark of primary TTP [4]. Synthesized vWF is assembled in ultralarge multimers (ULvWF) in plasma, then degraded by ADAMTS13 into normal-size multimers mediated by platelet adhesion. Nevertheless, attenuation or deprivation of ADAMTS13 could inhibit it to induce platelet aggregation/consumption, which further causes the extensive thrombosis and thrombocytopenia [5].

Postmortem studies have shown that the frequency of cardiac involvement in TTP is greater than 50\% [6]. A recent clinical trial disclosed that $\mathrm{vWF}$ correlated with arterial stenosis [7], whereas TTP manifested initially by MI has been rarely referred previously. About half of malignancy-associated TTP pertains to gastric carcinoma [8]. The unstable property of gastric carcinoma may evoke gene mutation and accelerate invasion. The widely accepted pathogenesis, albeit controversial, is the endothelium injury in the bone marrow by direct tumor invasion [5] that causes ULvWF release or ADAMTS13 reduction. Hence, in this case, the diagnosis of tumor-associated secondary TTP was made.

Since its introduction in the 1970s, plasma exchange remains the cornerstone of treatment via removing toxic substances, proaggregant substances, and/or antibodies directed against ADAMTS13. Plasma exchange should be started as soon as the diagnosis of TTP is established or even suspected [9]. Pharmaceutical therapy can confuse the issue because thrombolytics and anticoagulants trigger an amplified risk of bleeding [10]; although anticoagulant therapy seemed imperative for our patient, his history of cerebral hemorrhage implied higher susceptibility. Consequently, we cancelled antiplatelet agents; cardiac catheterization was not on the agenda either. For cancer-related TTP, the key point is the effective antitumor treatment against tumor load. The suggestion of antitumor treatment was raised by us; however, this couldn't be implemented, given the irreversible renal failure and other ominous signs.

\section{Conclusions}

This case should remind physicians confronted with specific cardiovascular or oncological cases to consider microangiopathy as a differential diagnosis in patients with any unexplained cardiac symptom or atypical presentation. Early diagnosis and treatment are helpful in decreasing the sequelae of this syndrome.

\section{Acknowledgements}

We express our gratitude to the patient and the support of his family for our study and case report.

\section{References}

1 Moschcowitz E: An acute febrile pleiochromic anemia with hyaline thrombosis of the terminal arterioles and capillaries: an undescribed disease. 1925. Mt Sinai J Med 2003;70: 352-355.

$>2$ Hee HH, Robert M, Nickolas J, et al: A rare cause of acute myocardial infarction: Thrombotic thrombocytopenic purpura. Int J Cardiol 2009; 1:e1-e2.

$>3$ Veyradier A, Obert B, Houllier A, et al: Specific von Willebrand factor-cleaving protease in thrombotic microangiopathies: a study of 111 cases. Blood 2001;98:1765-1772.
-4 Kremer Hovinga JA, Studt JD, Lammle B: The von Willebrand factor-cleaving protease (ADAMTS-13) and the diagnosis of thrombotic thrombocytopenic purpura (TTP). Pathophysiol Haemost Thromb 2003;33:417-421.

5 Chang J, Naqvi T: The thrombotic thrombocytopenic purpura associated with bone marrow metastasis and secondary myelofibrosis in cancer. Oncologist 2003;8:375-380.

-6 Ridolfi RL, Bell WR: Thrombotic thrombocytopenic purpura. Report of 25 cases and review of the literature. Medicine (Baltimore) 1981;60:413-428.
7 Yang H, Xu JX, Kong XZ, et al: Relations between plasma von Willebrand factor or endothelin-1 and restenosis following carotid artery stenting. Med Princ Pract 2012;21:538-542.

8 Murgo AJ: Thrombotic microangiopathy in the cancer patient including those induced by chemotherapeutic agents. Semin Hematol 1987;24:161-177.

-9 Coppo P, Veyradier A: Current management and therapeutical perspectives in thrombotic thrombocytopenic purpura. Presse Med 2012;41:e163-e176.

10 Muniz AE, Barbee RW: Thrombotic thrombocytopenic purpura (TTP) presenting as pancreatitis. J Emerg Med 2003;24:407-411. 